\title{
Operando electrochemical spectroscopic ellipsometry: Insights into electrochemical behavior of catalyst materials under realistic working conditions
}

\section{René Sachse}

Federal Institute for Materials Research and Testing (BAM)

Mario Sahre

Federal Institute for Materials Research and Testing (BAM)

Michael Bernicke

Technische Universität Berlin

Denis Bernsmeier

Technische Universität Berlin

\section{Vasile-Dan Hodoroaba}

Federal Institute for Materials Research and Testing (BAM)

\section{Peter Strasser}

Technical University of Berlin https://orcid.org/0000-0002-3884-436X

\section{Ralph Kraehnert}

Technical University Berlin

Andreas Hertwig ( $\nabla$ andreas.hertwig@bam.de)

Federal Institute for Materials Research and Testing (BAM)

\section{Article}

Keywords: operando spectroscopy, spectroscopic ellipsometry, oxygen evolution reaction, mesoporous iridium oxide films, platinum cyclic voltammetry

Posted Date: October 25th, 2021

DOl: https://doi.org/10.21203/rs.3.rs-934130/v1

License: (9) (1) This work is licensed under a Creative Commons Attribution 4.0 International License. Read Full License 


\title{
Operando electrochemical spectroscopic ellipsometry: Insights into electrochemical behavior of catalyst materials under realistic working conditions
}

René Sachse $e^{[a, b]}$, Mario Sahre ${ }^{[a]}$, Michael Bernicke $e^{[b]}$, Denis Bernsmeier ${ }^{[b]}$, Vasile-Dan Hodoroaba ${ }^{[a]}$, Peter Strasser ${ }^{[b]}$, Ralph Kraehnert* ${ }^{*}[\mathrm{~b}]$ and Andreas Hertwig* ${ }^{[a]}$

[a] Federal Institute for Materials Research and Testing (BAM), Unter den Eichen 44-46, 12203 Berlin, Germany

[b] Technische Universität Berlin, Faculty II Mathematics and Natural Sciences, Institute of Chemistry, Straße des 17. Juni 135, 10623 Berlin, Germany.

Corresponding Authors: *ralph.kraehnert@tu-berlin.de; *andreas.hertwig@bam.de

Keywords: operando spectroscopy, spectroscopic ellipsometry, oxygen evolution reaction, mesoporous iridium oxide films, platinum cyclic voltammetry

\begin{abstract}
Large-scale hydrogen production using proton exchange membrane water electrolysis (PEM-EL) requires a drastic reduction in the current noble metal content of the electrodes. To achieve this, new analytical techniques for characterizing solid-liquid catalytic interfaces, applicable in operando under a variety of realistic operating conditions, are critical because they provide a deeper understanding of the relationships between catalyst state, structure, and catalytic performance. However, many analytical techniques cannot be applied in liquid environments at realistic potentials and current densities.

We propose electrochemical spectroscopic ellipsometric analysis (ECSE), performed in realistic electrolysis cells, as a new analytical operando method for characterizing electrochemical reactions at solid-liquid interfaces under widely varying working conditions. The method is first validated on a platinum surface using ex-situ and operando ellipsometric analyses. Subsequently, the physicochemistry of a mesoporous $\mathrm{IrO}_{x}$ catalyst film under oxygen evolution reaction (OER) potentials was investigated, showing precise reversible and irreversible potential-dependent variations of a number of physical material properties relevant to catalysis.
\end{abstract}




\section{Main article}

\section{Introduction}

Due to the demand for energy with a low carbon dioxide footprint, efforts to develop sustainable and fossil-free energy sources are of great importance. ${ }^{1}$ Hydrogen can act as a renewable energy carrier and as a resource for chemical processes.,3 Green hydrogen is produced by electrolysis using renewable energy. Catalysts play an important role by increasing the rate, efficiency, and selectivity of chemical reactions. In water electrolysis, limiting factors are the not well understood reaction mechanism in the oxygen evolution reaction (OER) and the use of expensive materials, e.g. iridium for acidic water splitting. ${ }^{4-6}$

Significant progress has been made recently in the understanding of electrocatalysts, particularly in water splitting, i.e., both the OER and the hydrogen evolution reaction (HER). The combination of theoretical and experimental studies has led to a new understanding of catalytic trends enabling the development of more efficient electrocatalysts. ${ }^{4}$ In situ and/or operando experiments allow the catalytic activity to be analyzed under specific/realistic working conditions during the reaction. Often, these methods are based on X-rays, performed at synchrotron facilities in ultra-high vacuum with specially developed measurement setups and cells. ${ }^{7}$

A strong correlation of iridium hydroxo (Ir-OH) surface species with the OER activity was demonstrated using operando X-ray absorption spectroscopy (XAS) and X-ray photoelectron spectroscopy (XPS) on iridium oxide powder materials, observing a mixture of $\left|r^{\prime \prime \prime} /\right|^{\prime l V}$ oxidation states transitioning to higher oxidation states above $1.00 \mathrm{~V}$ vs. RHE. ${ }^{8} \mathrm{~A}$ decrease in intrinsic catalyst activity, was attributed to the presence of different $\mathrm{IrO}_{2}$ facets. The authors also suggested that gas bubbles are trapped in the mesopores of the sample blocking parts of the active sites. Direct analytical evidence of these assumptions was not given.

The development of vacuum-free experiments promises easier access to key parameters related to electrochemical activity of electrocatalysts. Spectroscopic ellipsometry (SE) enables a vacuum-free and non-destructive analysis, as it can be used under different environmental conditions. A classical application of ellipsometry is the study of layers on top of semiconductor materials. ${ }^{9}$ Further development of spectroscopic ellipsometric measurements allows the in-situ study of interfaces during layer growth, adsorption/desorption of solvents, or biomolecular processes. ${ }^{10-13}$ Ellipsometry is based on determining the change in the polarization state of an electromagnetic wave upon reflection, often quantified in the ellipsometric quantities $\Psi$ and $\Delta$, which are related to an amplitude ratio and a phase shift, respectively. In-situ ellipsometry is often used to study film growth in coating technology. ${ }^{10}$ It can be used to control film thickness during deposition and to study film growth rate, e.g., in atomic layer deposition (ALD).

SE can also be used for electrochemical processes. First ex-situ investigations and modeling of mesoporous iridium oxide films used in acidic OER showed accurate results of material specific parameters such as film thickness, porosity and resistivity compared to other methods. Electronic properties could be derived and correlated with activities in the OER. ${ }^{14}$ 
Here, we report on the analysis of electrocatalytic surfaces by operando electrochemical spectroscopic ellipsometry (ECSE). The setup consists of a spectroscopic ellipsometer and an electrochemical flow cell providing a realistic sample environment suitable for spectroscopy (Scheme 1). The setup was validated by a quantitative time-resolved analysis of reaction-induced surface oxidation and reduction of a bulk polycrystalline platinum surface. With this setup, we studied highly active $1 \mathrm{rO} \mathrm{x}_{\mathrm{x}}$-based porous model catalysts to track the catalyst surface and the evolved gas during dynamic OER conditions. The ellipsometric model provides time- and potential-dependent information of properties such as resistivity, interband transition energies, and the degree of pore filling with gas.
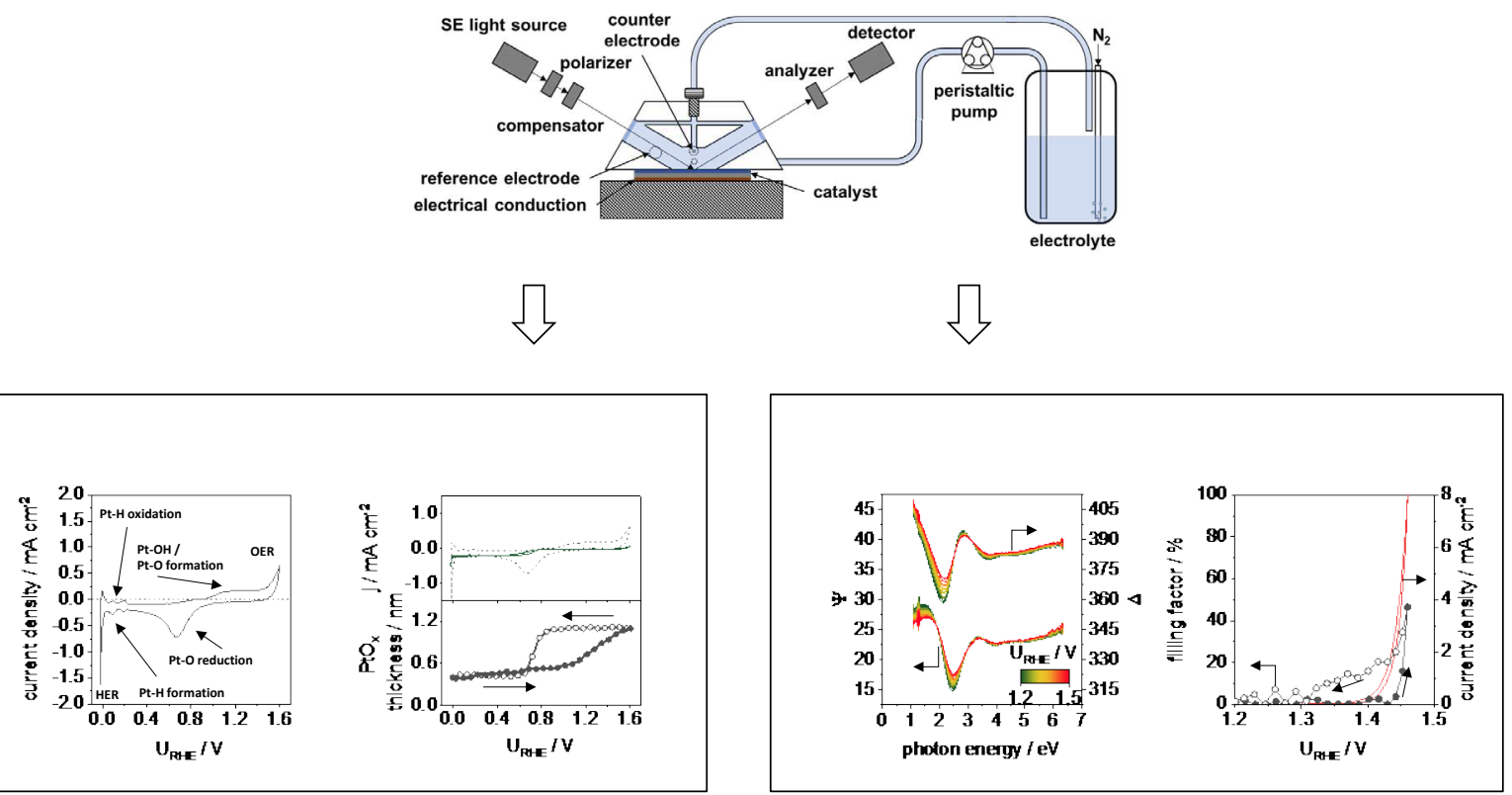

Scheme 1: Illustration of the developed operando electrochemical spectroscopic ellipsometry (ECSE) approach for the analysis of material properties under realistic working conditions. The approach includes the validation of a quantitative time-resolved analysis of reaction-induced oxidation and reduction of a Pt surface and the application for a time- and potential-dependent analysis of material properties of a mesoporous IrO $_{x}$ film during dynamic OER measurements. 


\section{Electrochemical, ex-situ SE and ECSE analysis of platinum}

Cyclic voltammetry on a platinum surface is used to validate the ECSE method. Cyclic voltammograms were recorded in a standard three-electrode setup as well as in the flow cell setup, (see the supporting information SI1). The electrolyte was $0.1 \mathrm{M} \mathrm{HClO}_{4}$ and a scan rate of $100 \mathrm{mV} \mathrm{s}^{-1}$ was applied. The resulting $\mathrm{Pt} \mathrm{CV}$ in the standard three-electrode configuration illustrates the in literature reported processes of the $\mathrm{Pt}-\mathrm{H}$ formation and oxidation, the double-layer region as well as the $\mathrm{Pt}-\mathrm{O}$ formation and reduction contributing to the characteristic shape..$^{15}$ The measurement in the flow cell setup exhibits the same characteristic processes and shape, with slightly lower signal intensity (current density response).

For the study of materials in ECSE, it is useful to study the materials in advance in an ex-situ analysis and develop a parameterization of the dielectric function. As a result, the resistivity at the surface (Pt/PtOx/air interface) with a value of $1.20 \cdot 10^{-5} \mathrm{Ohm} \mathrm{cm}$ and a thickness of about $0.33 \pm 0.26 \mathrm{~nm}$ for the $\mathrm{PtO}_{x}$ layer were obtained and are shown in the supporting information SI2.

The operando investigations of Pt using the ECSE flow cell setup are described in supporting information SI3 in detail. The ellipsometric measurement was corrected for the window and electrolyte effect using a Si wafer with a known oxide layer thickness (see supporting information SI4). ECSE was measured between $-0.28 \mathrm{~V}$ and $1.35 \mathrm{~V}$ against an $\mathrm{Ag} / \mathrm{AgCl}$ reference electrode $(+0.256 \mathrm{~V}$ vs. $\mathrm{RHE}$ at $\mathrm{pH} 1)$, a Pt wire as counter electrode and a cycle speed of $2 \mathrm{mV} \mathrm{s}^{-1}$. The model derived from the ex-situ Pt analysis (see supporting information SI2) was used together with the dielectric function of the electrolyte which served as the ambient above the Pt surface. Figure 1 presents the results of the electrochemical and spectroscopic ellipsometric measurements of the polycrystalline platinum surface. 


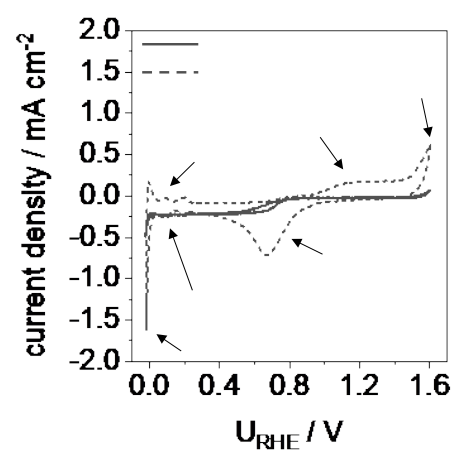

b)
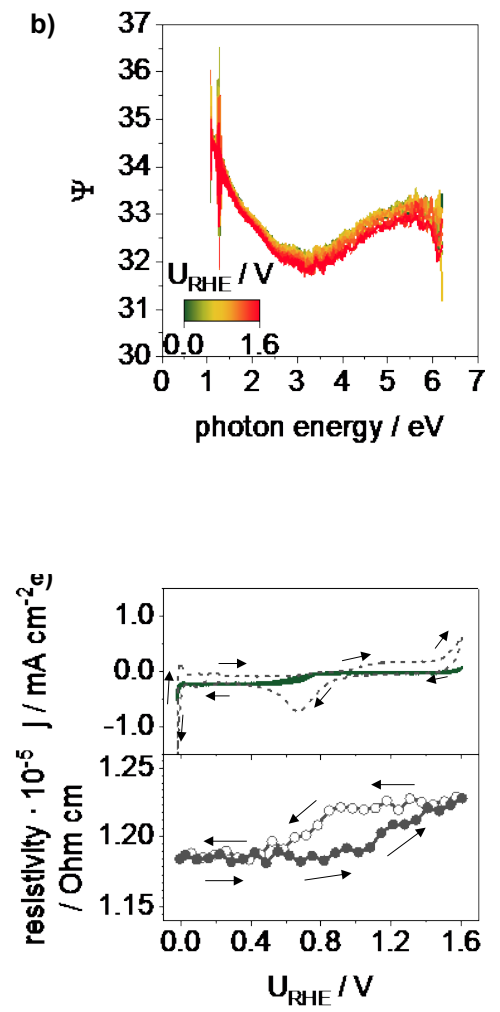

c)
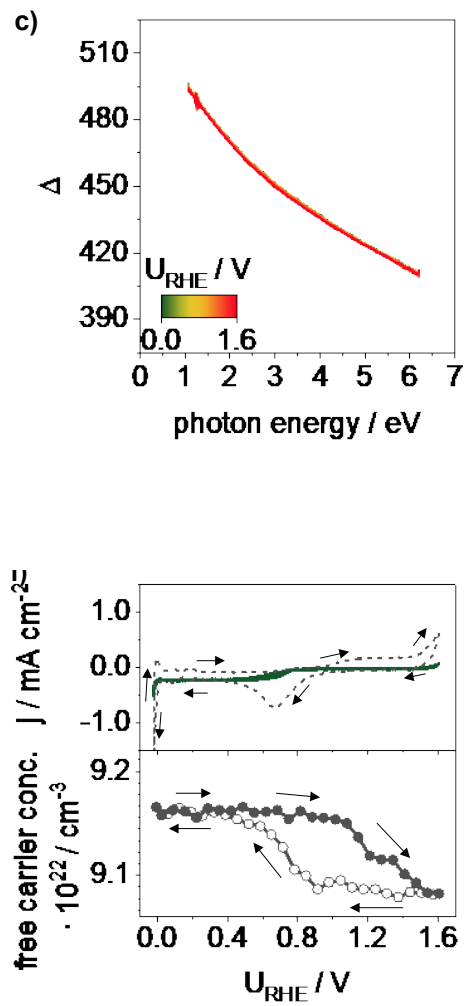

Figure 1: Operando ellipsometric investigations during cyclic voltammetry of bulk platinum. a) cyclovoltammetry in a potential range of $-0.28 \mathrm{~V}$ and $1.35 \mathrm{~V}$ against an $\mathrm{Ag} / \mathrm{AgCl}$ reference electrode $(+0.256 \mathrm{~V}$ vs. $\mathrm{RHE}$ at pH 1$)$ in $\mathrm{N}_{2-}$ purged $0.1 \mathrm{M} \mathrm{HClO}_{4}$, a flow rate of $85 \mathrm{ml} \mathrm{min}^{-1}$ and a cycle speed of $100 \mathrm{mV} \mathrm{s}^{-1}$ and $2 \mathrm{mV} \mathrm{s}^{-1}$, respectively. b) $\Psi$ spectra and c) $\Delta$ spectra of the measured platinum at different potentials. d) dependence of the $\mathrm{PtO}_{x}$ layer thickness on the applied potential. e) Pt resistivity as a function of the potential. f) changes of free carrier concentration during cyclic voltammetry. CV measurements of $100 \mathrm{mV} \mathrm{s}^{-1}$ and $2 \mathrm{mV} \mathrm{s}^{-1}$ in d) - f) are shown for the comparison of the model results with the electrochemical oxidation and reduction of the Pt surface.

The shape of the cyclic Pt voltammogram at a reduced scan rate of $2 \mathrm{mV} \mathrm{s}^{-1}$ (solid line) differs from the $\mathrm{CV}$ at a scan rate of $100 \mathrm{mV} \mathrm{s}^{-1}$ (dashed line), which is due to the dependence of the current response on the scan rate (Figure 1a). ${ }^{16}$ However, the characteristic HER and OER regimes as well as a change of the current density in the range of $0.6 \mathrm{~V}_{\mathrm{RHE}}$ are clearly visible, which probably indicates the Pt-O formation and/or reduction (oxygen chemisorption).

The corresponding $\Psi$ and $\Delta$ spectra (Figure $1 \mathrm{~b}$ and $\mathrm{c}$ ) show slight changes during the cyclic voltammetry measurement. For a better visualization of the changes, the spectra are plotted at selected energies with their fit results against the acquisition time (see supporting information SI5). From the fit, we obtain changes in the dielectric functions (see supporting information SI5), the $\mathrm{PtO}_{\mathrm{x}}$ layer thickness and electrical properties of the Pt layer (substrate). The CV curves with scan rates of $2 \mathrm{mV} \mathrm{s}^{-1}$ and $100 \mathrm{mV} \mathrm{s}^{-1}$ show the potential-dependent changes.

Figure 1d displays the $\mathrm{PtO}_{\mathrm{x}}$ layer thickness as a function of the potential, starting with the thickness of about $0.33 \mathrm{~nm}$ as obtained from the ex-situ SE characterization. By scanning from about $0.0 \mathrm{~V}_{\mathrm{RHE}}$ towards higher potentials (black circles), a roughly constant thickness is observed until a potential of $0.6 \mathrm{~V}_{\mathrm{RHE}}$, followed by a slight increase up to $1.1 \mathrm{~V}_{\mathrm{RHE}}$. At potentials higher than $1.1 \mathrm{~V}_{\mathrm{RHE}}$, the $\mathrm{PtO}_{\mathrm{x}}$ layer 
thickness increases to $1.1 \mathrm{~nm}$ in the OER regime (1.6 $\left.\mathrm{V}_{\mathrm{RHE}}\right)$. In the back-scan towards lower potentials (empty circles), the thickness does not change until a potential of $0.8 \mathrm{~V}_{\mathrm{RHE}}$ is reached. Subsequently, a decrease occurs until a potential of about $0.6 \mathrm{VRHE}$ and a thickness of about $0.33 \mathrm{~nm}$. Below this potential (0.6 $\mathrm{V}_{\mathrm{RHE}}$ ) the $\mathrm{PtO}_{\mathrm{x}}$ thickness remains relatively constant at $0.33 \mathrm{~nm}$ until the $\mathrm{HER}$ regime is reached. This hysteresic behavior agree well with the previously described oxidation/reduction mechanism of $\mathrm{Pt}$ during cyclovoltammetry.

Oxide layers on platinum of up to $1.0 \mathrm{~nm}$ were previously determined ellipsometrically. ${ }^{17}$ Investigations of the formation and growth of oxide films on platinum electrodes showed that the growth rate can be up to $1.2 \mathrm{~nm} \mathrm{~min}{ }^{-1}$ at an oxidative potential of $1.20 \mathrm{~V}_{\mathrm{Ag} / \mathrm{AgCl}}\left(\sim 1.46 \mathrm{~V}_{\mathrm{RHE}}\right){ }^{18} \mathrm{In}$ addition, Erbe and coworkers demonstrated the reduction and growth of a thin oxide layer of approximately $0.1 \mathrm{~nm}-2.1 \mathrm{~nm}$ during dynamic potential scanning in an in-situ ellipsometric study on the potentiostatic treatment of zinc, showing similar results as the deduced $\mathrm{PtO}_{x}$ layer growth on $\mathrm{Pt} .{ }^{19}$

The resistivity of the electrode's surface/interface shows a similar behavior as the thickness of the $\mathrm{PtO}_{x}$ layer and seems to depend on its growth rate (Figure 1e). This means that the resistivity has a constant value up to a potential of $1.1 \mathrm{~V}_{\mathrm{RHE}}$ and then increases as the $\mathrm{PtO}_{\mathrm{x}}$ layer grows. In the back scan up to a potential of about $0.8 \mathrm{~V} \mathrm{RHE}$, the resistivity is unchanged and returns to the initial value at lower potentials. It should be noted that the change of the resistivity takes place in a very small range of about $4.5 \cdot 10^{-}$ ${ }^{7} \mathrm{Ohm} \mathrm{cm}$.

Free carrier concentration values can be calculated from the dielectric function (see supporting information SI2). The free carrier concentration is displayed in Figure $1 \mathrm{f}$ and also shows a hysteresis behavior. However, the free carrier concentration decreases with an increasing of the $\mathrm{PtO}_{\mathrm{x}}$ layer. Due to the electrophilic nature of the oxygen ligands and the formation of Pt-O bonds, there is probably an interaction from the Pt electrons with the oxygen species. This may lead to a decrease in free carrier concentration and the observed increase in resistivity. Also, in this case the changes of the free carrier concentration are very small. 


\section{ECSE investigation of a mesoporous $\operatorname{IrO}$ film calcined at $375^{\circ} \mathrm{C}$}

For modeling of mesoporous iridium oxide films during electrochemical investigations, a modified model as described in ref. 14 was used. The model consists of a Ti substrate layer (multi-peak model), a $\mathrm{TiO}_{\mathrm{x}}$ layer (single Tauc-Lorentz oscillator), and a mesoporous $\mathrm{IrO}_{x}$ layer. The latter was modeled by an a-BEMA parameterization with an additional isotropic BEMA for the volume fractions of gas (air/void) and electrolyte within the pores instead of the air/void as described in ref. 14. In addition, the electrolyte was used as the ambient layer above the mesoporous $1 \mathrm{rO}_{\mathrm{x}}$ layer and its parameters obtained from the calibration procedure (see supporting information SI4).

The polished Ti substrate was analyzed before dip-coating and after film deposition and calcination. The thickness of the $\mathrm{TiO}_{\mathrm{x}}$ layer was determined before $(2.6 \mathrm{~nm} \pm 0.5 \mathrm{~nm})$ and after the calcination procedure (uncoated area: $3.4 \mathrm{~nm} \pm 1.9 \mathrm{~nm}$; below IrOx layer: $2.7 \mathrm{~nm} \pm 0.4 \mathrm{~nm}$ ) and was fixed for the ECSE analysis, as were the parameters of the Ti substrate.

Assuming that during electrochemistry the material properties as well as the interactions between iridium and oxygen change with the applied potential, the resistivity $\left(\rho_{R}\right)$ and mean scattering time $(\tau)$ of the Drude function, the amplitude (Amp) and full width at half-maximum $(\Gamma)$ of each Lorentz oscillator as well as the volume fraction of the produced gas within the pores were used as free parameters (see supporting information SI2). The center energy values of each Lorentz oscillator were fixed during the ECSE investigations after the ex-situ modeling of these values. It was also assumed that the film thickness and pore anisotropy (geometry) are not affected by electrochemical investigation. To prove the independence of the $\mathrm{IrO}_{x}$ layer thickness from potential changes, as determined for the $\mathrm{PtO}_{\mathrm{x}}$ film, XRR measurements were performed to determine the film thickness at different ambient humidity conditions (between $16 \%$ and nearly $100 \%$, see supporting information SI6).

The mesoporous $\mathrm{IrO}_{x}$ film, calcined at $375^{\circ} \mathrm{C}$, was investigated ellipsometrically under electrochemical conditions using cyclic voltammetry in a potential range of 1.20 $\mathrm{V}_{\mathrm{RHE}}$ and $1.48 \mathrm{~V}_{\mathrm{RHE}}$ with a scan rate of $1 \mathrm{mV} \mathrm{s}^{-1}$. A platinum wire was used as counter electrode and an $\mathrm{Ag} / \mathrm{AgCl}$ as reference electrode $(+0.256 \mathrm{~V}$ vs. $\mathrm{RHE}$ at $\mathrm{pH} 1)$. $\mathrm{N}_{2}$-purged $0.1 \mathrm{M} \mathrm{HClO}_{4}$ was used as electrolyte with a constant flow rate of $85 \mathrm{ml} \mathrm{min}^{-1}$. 
Figure 2 presents the cyclic voltammetry measurement in the OER region (a), $\Psi$ (b) and $\Delta$ (c) spectra at selected photon energies as a function of the measurement time, $d)$ surface $\left(-\operatorname{Im}\left((1+\varepsilon)^{-1}\right)\right)$ VEELS spectra, $e$ ) the center energy values of the identified peaks for surface VEELS as well as $f$ ) the intraband transition energies as a function of the potential of the mesoporous $1 \mathrm{rO}_{\mathrm{x}}$ film calcined at $375^{\circ} \mathrm{C}$.
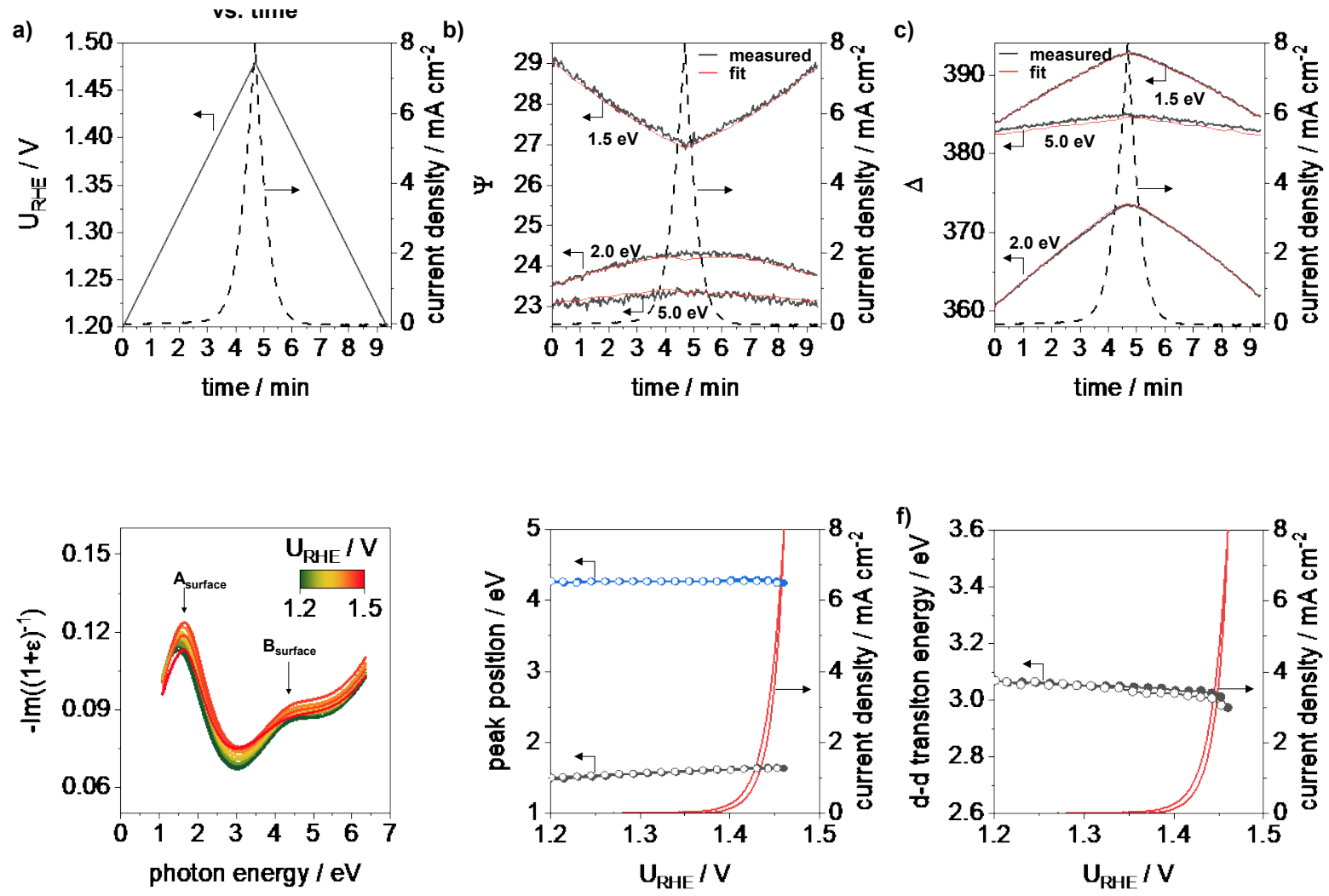

Figure 2: Operando ECSE investigations in the OER regime of the mesoporous $1 \mathrm{rO} \mathrm{O}_{x}$ film calcined at $375^{\circ} \mathrm{C}$. a) potential and current density as a function of acquisition time. The cyclovoltammetry was performed in a potential range of $1.20 \mathrm{~V}_{\mathrm{RHE}}$ and $1.48 \mathrm{~V}_{\mathrm{RHE}}$ in $\mathrm{N}_{2}$ purged $0.1 \mathrm{M} \mathrm{HClO}_{4}$ with a scan rate of $1 \mathrm{mV} \mathrm{s}^{-1}$ and an electrolyte flow rate of $85 \mathrm{ml} \mathrm{min}^{-1}$. Potentials were measured against an $\mathrm{Ag} / \mathrm{AgCl}$ electrode, which referred to $\mathrm{RHE}(+0.256 \mathrm{~V}$ at $\mathrm{pH} 1)$. b) $\Psi$ and c) $\Delta$ spectra with fit results at photon energies of $1.5 \mathrm{eV}, 2.0 \mathrm{eV}$ and $5.0 \mathrm{eV}$ together with the current density curve as a function of acquisition time. d) calculated surface VEEL spectra at different potentials during OER. e) A and B peaks of surface VEELS as a function of the applied potential. f) derived intraband transition energies between the Ir $5 d t_{2 g}$ and $e_{g}$ band in dependence on the potential.

Cyclovoltammetry measurements of the catalyst film (Figure 2a) show a typical progress of the current response with increasing and decreasing potential for a mesoporous $\mathrm{IrO}$ x film reported in literature (see refs. 14,20). To address changes of the ellipsometric quantities $\Psi$ and $\Delta$ to the electrochemical behavior, the spectra at exemplary photon energies of $1.5 \mathrm{eV}, 2.0 \mathrm{eV}$ and $5.0 \mathrm{eV}$ (black curves) are plotted together with the current response (dashed line) against the acquisition time (Figure 2b, c). The largest changes in both spectra are observed in the low photon energy range $(<3 \mathrm{eV})$, where electronic effects occur. The spectra show characteristic changes in their curvature which are mostly reversible. However, especially in the low photon energy range, the spectra are not completely reversible and show a slight offset, with the initial value not being reached again at the end of the cycle (see e.g., $\Psi$ vs. time at $1.5 \mathrm{eV}$ or $\Delta$ vs. time at $2.0 \mathrm{eV}$ ). Nevertheless, the modeled spectra (red curves) are in good agreement with the measured $\Psi$ and $\Delta$ spectra. 
The dielectric function obtained by fitting can be converted into a valence electron energy loss spectrum (see supporting information SI7) as described in ref. 14. This allows a more detailed analysis of the electronic structure of the $\mathrm{IrO}_{x}$ material (matrix) under electrochemical working conditions. Figure $2 \mathrm{~d}$ shows the surface $\left(-\operatorname{Im}\left((1+\varepsilon)^{-1}\right)\right)$ VEELS spectra which have two features, here referred to as $A$ and B peaks. ${ }^{21-23}$ These features can be assigned to transition energies from the $O 2 p$ orbital to the partial occupied (A peak) and unoccupied (B peak) Ir $5 \mathrm{~d} \mathrm{t}_{2 g}$ orbital. A slight shift of the center energy to higher energies is observed especially for the $A$ peaks as well as a change in intensity for both $A$ and $B$ peaks.

Figure 2e displays the center energy of the $A$ and $B$ peak derived from the surface VEELS together with the current density curve as a function of the potential. The $A_{\text {surface }}$ peak indicates a mostly linear shift of the center energy with an increase in potential. The maximum energy value is reached at a potential of about 1.44 $\mathrm{V}_{\mathrm{RHE}}$ and then remains approximately constant. In contrast, the Bsurface peak demonstrates an almost constant energy value. The center energies of the two peaks in the bulk VEELS show a similar behavior, shifted by about $0.1 \mathrm{eV}$ to higher energies (supporting information SI7).

Based on crystal field theory, the Ir $5 \mathrm{~d}$ orbital splits into $t_{2 g}$ and $e_{g}$ sub-levels. ${ }^{24}$ From the Tauc relation as described in ref. 14 , the $d-d$ intraband transition energies for the $\operatorname{lr} 5 d t_{2 g}$ and $e_{g}$ sub-level splitting can be derived. These transition energies (Figure 2f) decrease linearly with increasing potential (black circles). A stronger decrease of the transition energy in the range of $1.44 \mathrm{~V}_{\mathrm{RHE}}$ is observed. In the back scan (white circles) slightly lower energy values until a potential of about $1.30 V_{R H E}$ are observed. 
Additionally, electrical parameters such as resistivity, free carrier concentration as well as the volume fraction of the produced gas (filling factor) were obtained. Figure 3 displays a) the filling factor, b) the resistivity, and c) the free carrier concentration as a function of the potential.
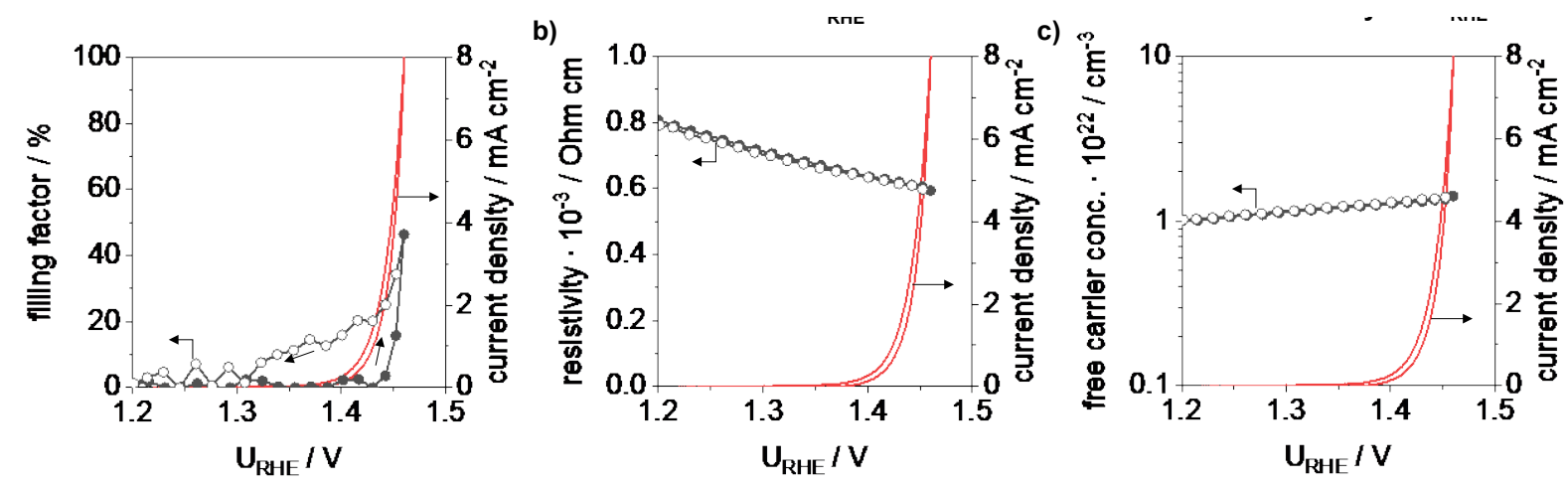

Figure 3: Derived properties from the model analysis during ECSE measurements in the OER regime. a) volume fraction of the produced gas (filling factor), b) resistivity, and c) free carrier concentration as a function of the applied potential.

The filling factor indicates a constant value close to zero for the forward-scanning cycle (black circles) until a potential of about $1.45 \mathrm{~V} \mathrm{VHE}$ and a current density of $2 \mathrm{~mA} \mathrm{~cm}-2$ are reached. As the potential continues to increase, the filling factor increases rapidly to a value of about $46 \%$. In the back-scanning cycle (white circles), the filling factor decreases more slowly than in the forwards cycle and reaches a value close to zero at a potential of about 1.30 VRHE. The model analysis thus indicates that the gas adheres to the pores surface and is slowly replaced by the electrolyte.

The resistivity values are completely reversible and decrease with increasing potential. This trend matches with the a constant to slightly increasing conductivity at low-potentials and a stronger increase at a potential of about $1.20 \mathrm{~V}_{\mathrm{RHE}}$ observed earlier by the group of Haverkamp. ${ }^{25}$ The assumption that the changes in the ellipsometric spectra are caused by changes in resistivity has to be validated. Therefore, operando ECSE measurements were performed on a non-conductive mesoporous titanium oxide ( $\mathrm{TiO}_{\mathrm{x}}$ ) film in the OER regime and in the potential range between $0.40 \mathrm{~V}_{\mathrm{RHE}}$ and $1.40 \mathrm{~V}_{\mathrm{RHE}}$. In both potential regions, no changes in the $\Psi$ and $\Delta$ spectra with changes in potential were observed (see supporting information SI8). The free carrier concentration of the $\mathrm{IrO}_{x}$ layer (Figure 3c) increases reversibly with increasing potential. 


\section{Discussion of the potential dependent changes of the $\mathrm{IrO}_{\mathrm{x}}$ material properties during OER}

The changes in the electrical and electronic properties of the $\mathrm{IrO}_{x}$ film during the OER as described above can probably be explained by changes in the band structure. In their work, Mortimer and coworkers describe electron transfer processes that can occur at the electrode-electrolyte interface ${ }^{26}$ In this process, the probability of electron transfer increases when the Fermi level increases to a level that facilitates charge transfer to an acceptor or oxidized state, or decreases to a level that facilitates charge transfer from a donor or reduced state. ${ }^{26}$ When an anodic potential is applied, the Fermi level decreases and reaches an energy level where electrons can be transferred from the donors in the electrolyte to the metal, increasing the density of states of the acceptors. ${ }^{26}$

In the case of the $\mathrm{IrO}_{x}$ film, the anodic potential could result in the $\mathrm{Ir} 5 \mathrm{~d} \mathrm{t}_{2 \mathrm{~g}}$ level being lowered. It has been reported that a shift of the Ir $5 d t_{2 g}$ level to lower energies causes an increase in the $p-d$ intermediate band transition energies, which can be explained by a higher electron density as well as a higher occupation of the d-band. ${ }^{27-29}$ Consequently, the covalent character of the $\mathrm{M}-\mathrm{O}$ bond is enhanced. ${ }^{27,28,30}$ The A peak in the VEEL spectra represents intermediate band transitions between the partially filled $t_{2 g}$ level of the $d$-band of iridium and the $p$-band of oxygen. Shifts in the interband transition energies are observed in the surface VEEL spectra (Figure 2e). A change in the transition between the p-bands of oxygen and the unoccupied $t_{2 g}$ bands of $\operatorname{Ir} 5 \mathrm{~d}$ is also evident in the B-peaks at higher potentials (Figure $2 e$ ). These changes indicate a shift in the $t_{2 g}$ band of Ir $5 d$. The change in free carrier concentration is also in good agreement with the increase in electron density with increasing covalency and overlap of the $p-d$ bands reported in the literature. ${ }^{27,28}$ The slight decrease in free carrier mobility (see supporting information SI7) may also indicate a stronger electron density due to the increased scattering of electrons and the associated energy change. ${ }^{32,34,35}$

A shift in the Ir $5 \mathrm{~d} \mathrm{t}_{2 \mathrm{~g}}$ level can also be observed during scanning toward cathodic potentials. A scan in the potential range between $0.40 \mathrm{~V}_{\mathrm{RHE}}-1.40 \mathrm{~V}_{\mathrm{RHE}}$ of the mesoporous $\mathrm{IrO}_{\mathrm{x}}$ film shows a significant change in the interband and intraband transition energies, as well as the transition of the Ir oxidation state (IIII//IIV redox pair) at about 0.95 VRHE (see supporting information SI9).

Finally, the adhesion of the produced gas should be discussed. The fraction of gas-filled versus electrolyte-filled pore volume (filling factor) shows a hysteresis loop (Figure 3a). This indicates that oxygen within the catalyst layer is only removed slowly. Stoerzinger et al. suggest this behavior in their studies on the contribution of lattice oxygen species from $\mathrm{RuO}_{2}$ films and nanoparticles to the oxygen gas using online electrochemical mass spectrometry (OLEMS). ${ }^{31}$ Therefore, it may be possible that diffusion limits gas dissolution and transport. However, since there is a complex interplay between the surface and pore properties, no definitive conclusion can be drawn with the present data and further detailed analysis has to be performed. 


\section{Conclusion}

With the ECSE approach, small changes on a platinum surface are detectable in cyclic voltammetry studies, such as oxidation and reduction of the time- and potential-dependent $\mathrm{PtO} \times$ layer growth and the associated changes in electrical properties. This fundamental study of operando ECSE measurement on a platinum surface demonstrates the accuracy of potential-dependent surface analysis and opens a wide field for electrochemical and electrocatalytic studies under operando conditions.

Operando ECSE analysis in the OER regime of a mesoporous $\mathrm{IrO}_{x}$ film, calcined at $375^{\circ} \mathrm{C}$, proves that the dielectric function changes due to the shift in the Ir oxidation states and the change of the $p-d$ interaction. A shift of the A peak in the VEEL spectra is visible, which was previously associated with a linear correlation of the intrinsic OER activity. The changes in the Ir $5 \mathrm{~d} \mathrm{t}_{2 g}$ band and the $p$-d interband transitions are also directly reflected in the electrical properties of the material. According to the current state of knowledge, the model analysis provides for the first time an analysis of the volume fraction of the produced gas and gives an indication of an accumulation of gas within the pores.

The analytical ECSE approach also provides the opportunity to study different materials under a variety of electrochemical processes to gain a deeper understanding of catalytic systems, batteries, and material corrosion. The ability to perform fast, vacuum-free, and non-destructive characterization under realistic potentials and current densities could also be used to study other electrochemical reactions such as the HER, HOR, or ORR in the future. 


\section{Methods}

\section{Chemicals}

For the synthesis of the mesoporous iridium oxide film iridium acetate $\left(\operatorname{Ir}\left(\mathrm{CH}_{3} \mathrm{COO}\right)_{n}, 99.95 \%\right.$ metals basis, ca. $48 \%$ Ir) was used from chemPUR and a triblock-copolymer (PEO-PB-PEO, containing 20400 $\mathrm{g} \mathrm{mol}^{-1}$ polyethylene oxide (PEO) and $10000 \mathrm{~g} \mathrm{~mol}^{-1}$ polybutadiene (PB)) was purchased from Polymer Service Merseburg $\mathrm{GmbH}^{32}$. Ethanol $(\mathrm{EtOH},>99 \%)$ was used as solvent from Sigma-Aldrich. All chemicals were used as received. A conductive titanium sheet $(25 \times 40 \mathrm{~mm})$ was used as substrate, which was polished using a suspension consisting of non-crystallizing colloidal silica suspension (200 ml; $0.25 \mu \mathrm{m}$; Struers, OP-S), hydrogen peroxide $(40 \mathrm{ml})$ and nitric acid $(1 \mathrm{ml})$, followed by a cleaning procedure with EtOH. The platinum foil for operando ECSE studies was polished using a $3 \mu \mathrm{m}$ diamond suspension (Struers, DiaDuo-2) and cleaned with ethanol.

\section{Synthesis of mesoporous metal oxide films}

The mesoporous iridium oxide film was synthesized according to the synthesis which is described by Ortel et al. ${ }^{33}$ In a slightly modified synthesis, $169 \mathrm{mg}$ of the PEO-PB-PEO polymer template were dissolved in $7.5 \mathrm{ml}$ ethanol at $40{ }^{\circ} \mathrm{C}$. After complete dissolution, $844 \mathrm{mg}$ iridium(III) acetate were added and the solution was stirred for $1 \mathrm{~h}$ at $40^{\circ} \mathrm{C}$. The dip-coating solution was transferred into a preheated Teflon cuvette $\left(2 \mathrm{~h}\right.$ at $\left.50^{\circ} \mathrm{C}\right)$ and dip-coating was immediately performed under controlled dip-coater conditions $\left(25^{\circ} \mathrm{C}, 40 \%\right.$ relative humidity) with a cuvette heater $\left(45^{\circ} \mathrm{C}\right)$ and a withdrawal rate of $200 \mathrm{~mm} \mathrm{~min}^{-1}$. As-synthesized films were dried for 5 minutes under the same conditions and subsequently calcined in a preheated muffle furnace in flowing air for 5 minutes at temperature of $375^{\circ} \mathrm{C}$.

The mesoporous titanium oxide film was synthesized according to the synthesis which is described by Ortel et al..$^{32}$ In a slight modified synthesis, $181 \mathrm{mg}$ of the PEO-PB-PEO polymer template were dissolved in $5 \mathrm{ml}$ ethanol at $40{ }^{\circ} \mathrm{C}$. In a second solution $1 \mathrm{~g} \mathrm{TiCl} 4$ was mixed with $5 \mathrm{ml}$ ethanol under an Ar atmosphere. After complete dissolution both solutions were mixed for $1 \mathrm{~h}$ at $40{ }^{\circ} \mathrm{C}$. Dip-coating was subsequently performed under the same conditions as described above.

\section{Physicochemical characterization}

SE measurements were performed with a variable angle spectroscopic ellipsometer M2000 DI (J.A. Woollam) in a spectral range between $0.7 \mathrm{eV}$ and $6.5 \mathrm{eV}(192 \mathrm{~nm}$ and $1697 \mathrm{~nm})$. Samples were measured ex-situ at angles of incidence of $65^{\circ}, 70^{\circ}$ and $75^{\circ}$ and acquisition times between $5 \mathrm{~s}$ and $10 \mathrm{~s}$. $\Psi$ and $\Delta$ spectra were analyzed with the software CompleteEASE (v. 6.42) using the models described above by using the model functions (see supporting information $\mathrm{SI}$ ).

Operando electrochemical spectroscopic ellipsometric analysis of all samples was performed with a flow cell setup in the ellipsometer described before (see supporting information $\mathrm{SI} 3$ ).

X-ray reflectometry $(\mathrm{XRR})$ were performed at a Bragg-Soller X-ray diffractometer system with a flat secondary monochromator and fixed Cu Ka-tube (Seifert XRD 3000TT). 


\section{Electrochemical testing}

Electrocatalytic measurements were performed using a Gamry interface 1000 (IFC1000) with the Gamry Framework Data Acquisition software (v. 7.07). The platinum sheet or coated titanium substrates served as working electrode, a platinum wire as counter electrode and an $\mathrm{Ag} / \mathrm{AgCl}(3 \mathrm{M} \mathrm{NaCl}$; flow cell type) as reference electrode. All potentials are recorded under room temperature and referred to the RHE. N2purged perchloric acid was used as electrolyte solution $\left(0.1 \mathrm{M} \mathrm{HClO}_{4}\right)$.

Platinum CVs were recorded in a standard three-electrode setup and in the flow cell setup in a potential range between $-0.28 \mathrm{~V}_{\mathrm{Ag} / \mathrm{AgCl}}$ and $1.35 \mathrm{~V}_{\mathrm{Ag} / \mathrm{AgCl}}(+0.256 \mathrm{~V}$ vs. RHE at $\mathrm{pH} 1)$ with a scan rate of $100 \mathrm{mV} \mathrm{s}^{-1}$ and $2 \mathrm{mV} \mathrm{s}^{-1}$ under operando conditions. Operando ECSE investigations of the metal oxide films were performed either in the OER regime $\left(1.20 \mathrm{~V}_{\mathrm{RHE}}-1.48 \mathrm{~V}_{\mathrm{RHE}}\right)$ or in a lower potential range (0.40 $\mathrm{V}_{\text {RHE }}$ and $1.40 \mathrm{~V}$ RHE) with scan rates of $1 \mathrm{mV} \mathrm{s}^{-1}$.

\section{Acknowledgment}

Andreas Hertwig and René Sachse acknowledge the project HyMET (16ENG03 Hybrid metrology for thin films in energy applications), which has received funding from the EMPIR programme co-financed by the Participating States and from the European Union's Horizon 2020 research and innovation programme. Denis Bernsmeier acknowledges generous funding from VIP+ (BMBF) under contract FKZ 03VP05390. Michael Bernicke and Ralph Kraehnert thank ATOKAT (BMBF, FKZ 03EK3052A). Ralph Kraehnert also thank DFG SPP 2080. Peter Strasser acknowledges partial funding by the Deutsche Forschungsgemeinschaft (DFG) through project STR 595/11-1 and by the Bundesministerium für Bildung und Forschung (BMBF) under grant „HyThroughGen”. 


\section{References}

1 Seh, Z. W. et al. Combining theory and experiment in electrocatalysis: Insights into materials design. Science 355, eaad4998, doi:10.1126/science.aad4998 (2017).

2 Olah, G. A., Goeppert, A. \& Prakash, G. S. Beyond oil and gas: the methanol economy. (John Wiley \& Sons, 2018).

3 Campos-Martin, J. M., Blanco-Brieva, G. \& Fierro, J. L. Hydrogen peroxide synthesis: an outlook beyond the anthraquinone process. Angewandte Chemie International Edition 45, 6962-6984 (2006).

4 Rossmeisl, J., Qu, Z. W., Zhu, H., Kroes, G. J. \& Nørskov, J. K. Electrolysis of water on oxide surfaces. J. Electroanal. Chem. 607, 83-89, doi:https://doi.org/10.1016/i.jelechem.2006.11.008 (2007).

5 Mavros, M. G. et al. What Can Density Functional Theory Tell Us about Artificial Catalytic Water Splitting? Inorganic Chemistry 53, 6386-6397, doi:10.1021/ic5002557 (2014).

Betley, T. A., Wu, Q., Van Voorhis, T. \& Nocera, D. G. Electronic Design Criteria for O-O Bond Formation via Metal-Oxo Complexes. Inorganic Chemistry 47, 1849-1861, doi:10.1021/ic701972n (2008).

7 Velasco-Vélez, J.-J. et al. A comparative study of electrochemical cells for in situ x-ray spectroscopies in the soft and tender x-ray range. Journal of Physics D: Applied Physics 54, 124003, doi:10.1088/1361-6463/abd2ed (2021).

8 Abbott, D. F. et al. Iridium Oxide for the Oxygen Evolution Reaction: Correlation between Particle Size, Morphology, and the Surface Hydroxo Layer from Operando XAS. Chemistry of Materials 28, 6591-6604, doi:10.1021/acs.chemmater.6b02625 (2016).

9 Hilfiker, J. N. et al. Survey of methods to characterize thin absorbing films with Spectroscopic Ellipsometry. Thin Solid Films 516, 7979-7989, doi:https://doi.org/10.1016/j.tsf.2008.04.060 (2008).

10 Aspnes, D. E. \& Theeten, J. B. Spectroscopic Analysis of the Interface Between Si and Its Thermally Grown Oxide. Journal of The Electrochemical Society 127, 1359-1365, doi:10.1149/1.2129899 (1980).

11 Langereis, E. et al. In situspectroscopic ellipsometry as a versatile tool for studying atomic layer deposition. Journal of Physics D: Applied Physics 42, 073001, doi:10.1088/00223727/42/7/073001 (2009).

12 Ohtsuka, T. \& Fushimi, K. Optical Characterization of Passive Oxides on Metals. Electrochemistry 84, 826-832, doi:10.5796/electrochemistry.84.826 (2016).

13 Kalas, B., Agocs, E., Romanenko, A. \& Petrik, P. In Situ Characterization of Biomaterials at Solid-Liquid Interfaces Using Ellipsometry in the UV-Visible-NIR Wavelength Range. physica status solidi (a) 216, 1800762, doi:https://doi.org/10.1002/pssa.201800762 (2019).

14 Sachse, R. et al. Assessing Optical and Electrical Properties of Highly Active IrOx Catalysts for the Electrochemical Oxygen Evolution Reaction via Spectroscopic Ellipsometry. ACS Catalysis 10, 14210-14223, doi:10.1021/acscatal.0c03800 (2020). 

nanostructured platinum electrodes - a cyclic voltammetry study. Physical Chemistry Chemical Physics 16, 8392-8399, doi:10.1039/C4CP00342J (2014).

Hamann, C. H., Hamnett, A. \& Vielstich, W. Electrochemistry. 2., completely rev. and updated ed. edn, (Wiley-VCH, 2007).

Gilroy, D. \& Conway, B. E. Surface oxidation and reduction of platinum electrodes: Coverage, kinetic and hysteresis studies. Canadian Journal of Chemistry 46, 875-890, doi:10.1139/v68149 (1968).

Percival, S. J. \& Zhang, B. Study of the Formation and Quick Growth of Thick Oxide Films Using Platinum Nanoelectrodes as a Model Electrocatalyst. Langmuir 30, 11235-11242, doi:10.1021/la502336e (2014).

Chen, Y. \& Erbe, A. In situ spectroscopic ellipsometry during electrochemical treatment of zinc in alkaline carbonate electrolyte. Surface Science 607, 39-46, doi:https://doi.org/10.1016/j.susc.2012.08.006 (2013).

Bernicke, M. et al. Iridium Oxide Coatings with Templated Porosity as Highly Active Oxygen Evolution Catalysts: Structure-Activity Relationships. ChemSusChem 8, 1908-1915, doi:doi:10.1002/cssc.201402988 (2015).

21 Goel, A. K., Skorinko, G. \& Pollak, F. H. Optical properties of single-crystal rutile Ru\$\{lmathrm\{O\}\}_\{2\}\$ and Ir $\left\{\{\mid m a t h r m\{O\}\} \_\{2\} \$\right.$ in the range 0.5 to $9.5 \mathrm{eV}$. Physical Review $B$ 24, 7342-7350, doi:10.1103/PhysRevB.24.7342 (1981).

Choi, W. S. et al. Dielectric constants of Ir, Ru, Pt, and IrO2: Contributions from bound charges. Physical Review B 74, 205117, doi:10.1103/PhysRevB.74.205117 (2006).

Mattheiss, L. F. Electronic structure of $\$\{\operatorname{lmathrm}\{\mathrm{RuO}\}\}_{-}\{2\} \$, \$\{\operatorname{lmathrm}\{\mathrm{O} \mathrm{O} O\}\} \_\{2\} \$$, and \$\{lmathrm\{|rO\}\}_\{2\}\$. Physical Review B 13, 2433-2450, doi:10.1103/PhysRevB.13.2433 (1976).

24 Pearson, R. G. Crystal field theory and substitution reactions of metal ions. Journal of Chemical Education 38, 164, doi:10.1021/ed038p164 (1961).

25 Ilyukhina, L., Sunde, S. \& Haverkamp, R. G. Electronic Structure and Growth of Electrochemically Formed Iridium Oxide Films. Journal of The Electrochemical Society 164, F1662-F1670 (2017).

Kumsa, D. W. et al. Electron transfer processes occurring on platinum neural stimulating electrodes: a tutorial on thei(Ve) profile. Journal of Neural Engineering 13, 052001, doi:10.1088/1741-2560/13/5/052001 (2016).

27 Sen, F. G. et al. Towards accurate prediction of catalytic activity in IrO2 nanoclusters via first principles-based variable charge force field. Journal of Materials Chemistry A 3, 18970-18982, doi:10.1039/C5TA04678E (2015).

28 Chen, J. et al. Low-Coordinate Iridium Oxide Confined on Graphitic Carbon Nitride for Highly Efficient Oxygen Evolution. Angewandte Chemie International Edition 58, 12540-12544, doi:https://doi.org/10.1002/anie.201907017 (2019).

29 Hwang, J. et al. Perovskites in catalysis and electrocatalysis. Science 358, 751-756, doi:10.1126/science.aam7092 (2017). 
30 Kuznetsov, D. A. et al. Tailoring Lattice Oxygen Binding in Ruthenium Pyrochlores to Enhance Oxygen Evolution Activity. Journal of the American Chemical Society 142, 7883-7888, doi:10.1021/jacs.0c01135 (2020).

31 Stoerzinger, K. A. et al. Orientation-Dependent Oxygen Evolution on RuO2 without Lattice Exchange. ACS Energy Letters 2, 876-881, doi:10.1021/acsenergylett.7b00135 (2017).

32 Ortel, E. et al. New Triblock Copolymer Templates, PEO-PB-PEO, for the Synthesis of Titania Films with Controlled Mesopore Size, Wall Thickness, and Bimodal Porosity. Small 8, 298-309, doi:doi:10.1002/smll.201101520 (2012).

33 Ortel, E., Reier, T., Strasser, P. \& Kraehnert, R. Mesoporous IrO2 Films Templated by PEOPB-PEO Block-Copolymers: Self-Assembly, Crystallization Behavior, and Electrocatalytic Performance. Chemistry of Materials 23, 3201-3209, doi:10.1021/cm200761f (2011). 


\section{Supplementary Files}

This is a list of supplementary files associated with this preprint. Click to download.

- SupplementaryInformation.docx 